\title{
Analysis and Evaluation of Structural Safety Inspection of a Railway Station
}

\author{
Guo Xiaoyan, Fang Lin, Ma Zefeng \\ Technical Center of East China Architectural Design Institute, Shanghai, China
}

\section{Email address:}

xiaoyan_guo@arcplus.com.cn (Guo Xiaoyan)

\section{To cite this article:}

Guo Xiaoyan, Fang Lin, Ma Zefeng. Analysis and Evaluation of Structural Safety Inspection of a Railway Station. Science Discovery. Vol. 8, No. 5, 2020, pp. 97-100. doi: 10.11648/j.sd.20200805.14

Received: August 31, 2020; Accepted: October 13, 2020; Published: October 17, 2020

\begin{abstract}
Based on the structural safety inspection of a railway station, first the current status and damage of the housing structure are introduced. It is analysed in this paper the safe capability of the railway station via precise on-site testing work. It is further discussed the influence of different modeling methods on the calculative results of the compressive bearing capacity of buttress column used in this masory structure. There is no software could accurately simulate the compressive bearing capacity of buttress column, for it is difficult to meet the actual needs of the project. This paper could be valuable future reference for the safety inspection and strengthening scheme of similar projects.
\end{abstract}

Keywords: Masory Structure, Structural Safty, Buttress Column

\section{某火车站结构安全性检测的分析与评估}

\section{郭晓燕, 方林, 马泽峰}

华东建筑设计研究院有限公司, 上海, 中国

\section{邮箱}

xiaoyan_guo@arcplus.com.cn（郭晓燕）

摘要: 以某火车站结构安全性检测为背景, 首先介绍了房屋的使用现状及使用过程中结构的损伤情况。通过现场检测, 对结构的安全性能进行了计算分析, 并对不同建模方式对砌体结构带壁柱墙的抗压承载力计算结果的影响进行了探讨。 对于带壁柱墙的计算目前还没有计算软件可以准确模拟, 各种建模方式均与实际计算结果存在误差, 难以满足实际工 程需要。本文建议如若粗略估算带壁柱墙的抗压承载力建议可采用扶壁柱位置纵墙变截面的方式建模。本文可为类似 工程的安全性鉴定与加固改造提供参考。

关键词: 砌体结构, 结构安全, 扶壁柱

\section{1. 工程概况}

某火车站始建于清光绪三十三年（1907年），是当时 上海地区唯一自备专用码头的铁路车站, 当时主要负责黄 浦江上货物的装卸, 车站在2009年关闭, 现已废弃。因建 造年份久远, 原设计相关图纸资料完全缺失。房屋建筑总
体平面布置如图1所示, 南侧为3幢砖墙承重的单层空旷房 屋, 中间为火车轨道, 北侧为站台, 平面形状大致呈矩形, 东西向长约 $173 \mathrm{~m}$, 南北向最宽处约 $25.4 \mathrm{~m}$ 。本文主要介绍 南侧 3 幢房屋的安全性检测。 


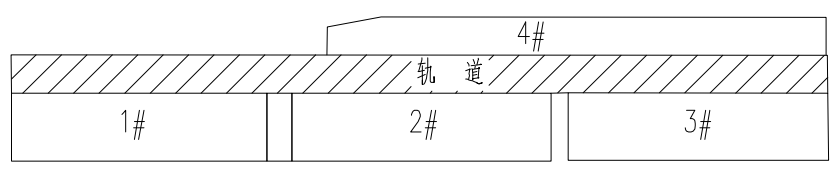

图1 房屋平面布局示意图。

1 号房东西向总长度为 $55.05 \mathrm{~m}$, 南北向总宽度为 $12.06 \mathrm{~m}$, 屋脊高度约为 $6.875 \mathrm{~m} ; 2$ 号房东西向总长度为 $55.16 \mathrm{~m}$, 南北向总宽度为 $12.07 \mathrm{~m}$, 屋脊高度约为 $6.745 \mathrm{~m}$; 3 号房东西向总长度为 $55.78 \mathrm{~m}$, 南北向总宽度为 $12 \mathrm{~m}$, 屋 脊高度约为 $6.78 \mathrm{~m}$ 。1 3号房屋面均采用坡屋面。图2、图3 为 1 号房实景照片。

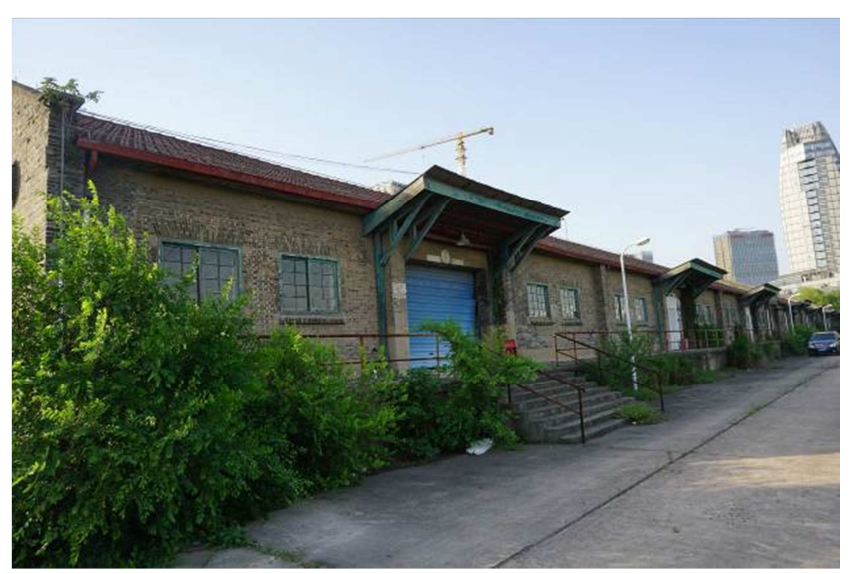

图2 1号房南立面。

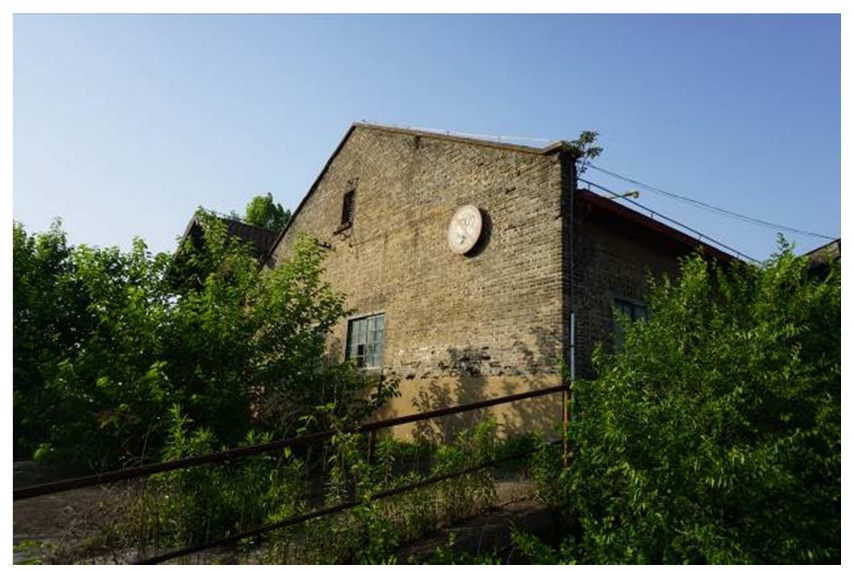

图3 1号房西立面。

\section{2. 房屋结构现况}

$1 、 2 、 3$ 号房均为砖墙承重的单层空旷房屋, 纵墙承 重, 承重墙采用青砖, 墙厚为 $340 \mathrm{~mm}$ (一砖半), 未设置 钢筋混凝土圈梁和构造柱。纵墙外不等距设置有扶壁柱, 突出墙面 $340 \mathrm{~mm}$ (一砖半)，1、2号房扶壁柱宽度 $450 \mathrm{~mm}$ （二砖），3号房扶壁柱宽度 $560 \mathrm{~mm}$ (二砖半）。屋顶采 用木屋架承重, 如图4所示, 基础均采用墙下条形基础。

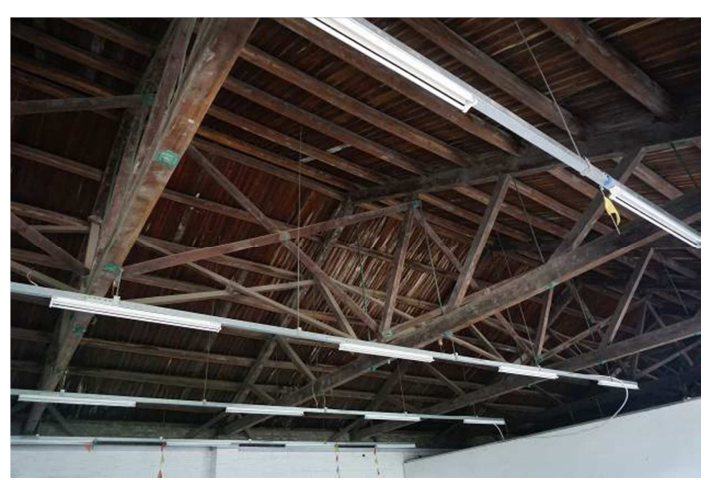

图41号房木屋架。

\section{3. 结构现状鉴定分析}

\section{1. 建筑结构布置和主要结构构件尺寸检测}

根据项目要求, 现场扫描人员, 对车站进行了全范围 三维激光扫描工作, 部分扫描结果如图5所示。根据现场 扫描结果, 将各站点云数据拼接, 初步形成房屋的整体三 维点云模型。点云模型可以真实反映房屋室内外的实际情 况, 为今后数据资料的调研提供便利, 更好地保护历史建 筑文化价值 $[4,5,7,8]$ 。

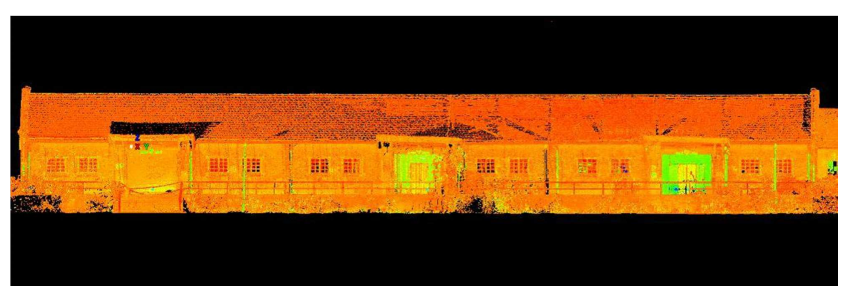

图5 1 号房南立面点云数据。

同时, 依托完整精确的车站点云模型, 可以对各个屋 架进行剖取, 进而对整体屋架形式及尺寸进行测量绘制, 尤其对于部分圆形的杆件采用点云尺寸自动拟合功能, 解 决了屋架圆形构件由于现场高度及测量方式等问题所产 生的技术难点, 如图6所示。

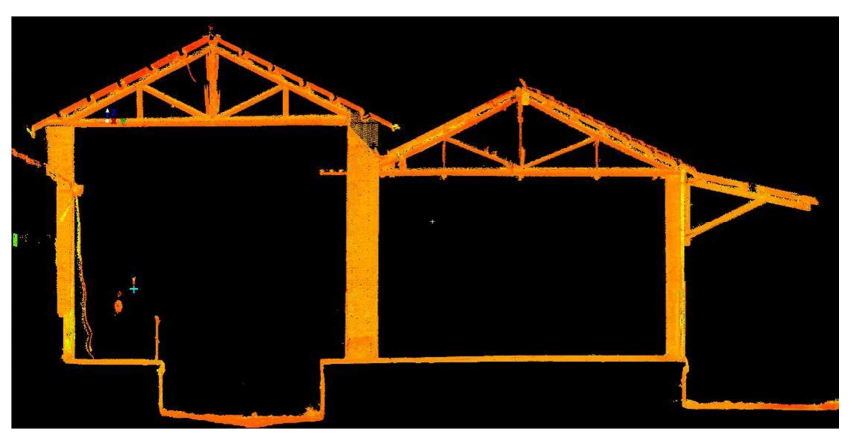

图6 屋架剖面点云数据。

\section{2. 结构材料检测}

现场对房屋砖、砂浆强度进行了抽样检测, 检测结果 表明: 1号房实测砖强度达到MU7.5, 砌筑砂浆达到M2.0; 2 号房实测砖强度达到MU4, 砌筑砂浆达到M1.5；3号房 
实测砖强度达到MU5, 砌筑砂浆达到M1.5[10]。损伤状况 调查结果表明, 房屋目前存在的主要为非结构性损伤, 主 要原因是由于年久失修、材料老化、受潮腐蚀、木材干裂 引起（图7） [1, 2,6,9]。

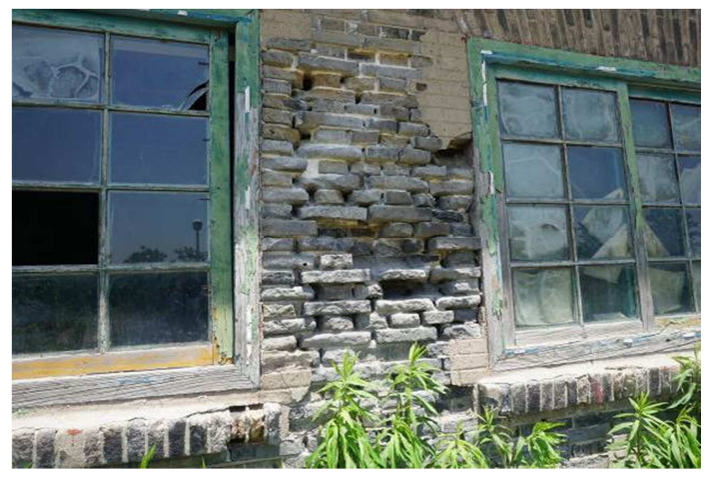

(a) 外墙局部破损

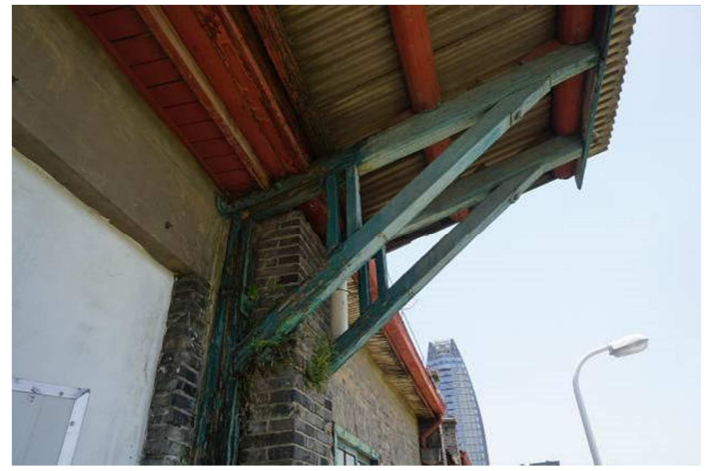

（b）雨蓬木构架腐蚀开裂

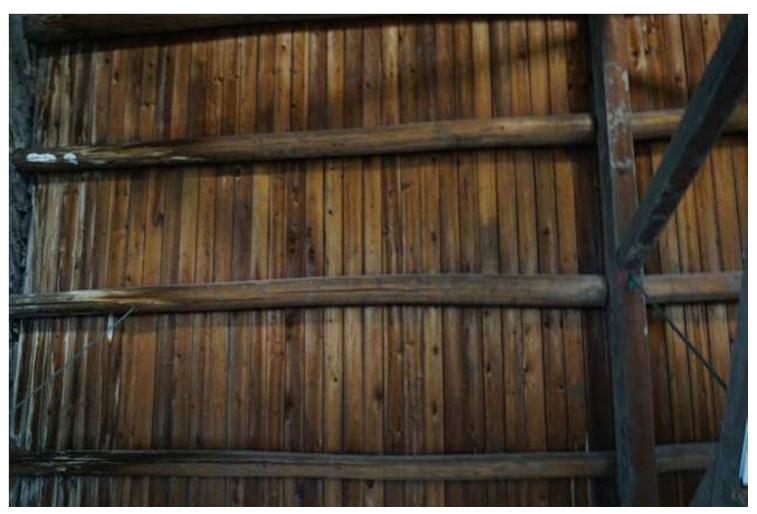

(c) 室内木檩条开裂较普遍

图7 房屋损伤状况。

\section{3. 结构安全性分析}

根据现场检测结果，结合房屋目前使用现状，对房屋 进行结构承载力验算, 计算分析采用中国建筑科学研究院 编制的PKPM建筑结构计算软件, 砖柱承载力采取手算复 核。

验算仅考虑正常使用荷载（静力荷载）作用下，不考 虑地震作用。风荷载基本风压为 $0.55 \mathrm{kN} / \mathrm{m}^{2}$, 地面粗䊁度 为 $\mathrm{C}$ 类。结果表明: 各房屋墙体受压承载力及高厚比、木 屋架、地基基础承载力满足规范要求。

\section{4. 工程思考}

本工程建筑形式规整, 传力路径明确, 属于常规结构 安全性检测项目。但由于建造年代久远, 目前的计算软件 主要针对新建建筑的验算比较准确, 针对早期砌体结构在 计算中存在的一些问题还需要检测人员仔细甄别判断。

\section{1. 计算软件的选择}

检测鉴定中通常使用的YJK和PKPM软件在砌体结构 和混凝土结构的计算中并没有太大的区别。但是在本项目 中, 实测砖强度和砂浆强度均较低, 低于现行国家标准《砌 体结构设计规范》GB50003-2011中3.2节所列的最低值[3]。 在计算过程中, YJK和PKPM虽然都可以输入实测强度, 但YJK会自动取表格中的最低值, 这样使得最终的计算结 果偏于不安全。

\section{2. 砌体结构带壁柱墙的抗压承载力的软件计算方法探 讨}

扶壁柱是早期砌体结构中常见的受力构件, 但在实际 计算过程中, 采用不同软件模拟方法, 其抗压承载力的计 算结果与相关设计规范的计算结果均有误差。在此, 以南 侧 1 号房为例, 对 1-2/1-A轴处带壁柱墙抗压承载力计算结 果进行探讨。

按照《砌体结构设计规范》GB50003-2011中规定对于 带壁柱墙按 $\mathrm{T}$ 形截面进行验算, 条文4.2.8第2条规定, 带壁 柱墙的计算翼缘宽度可取壁柱宽加 $2 / 3$ 墙高, 但不应大于 窗间墙宽度和相邻壁柱间的距离。按照规范 5.1 节中, 根 据以下公式进行验算:

$$
\begin{aligned}
& N \leq \varphi \mathrm{f} A \\
& \beta=\gamma_{\beta} \frac{H_{0}}{\mathrm{~h}_{T}}
\end{aligned}
$$

由于PKPM软件无法直接模拟带壁柱墙建模, 实际中, 考虑两种方法处理: 壁柱处加横墙建模, 壁柱处墙体采用 变截面墙体建模。根据以上两种方法, 计算结果与规范公 式计算结果的误差，前者为 $16 \%$, 后者为 $-6 \%$ 。分析其原 因, 前者在考虑壁柱处加横墙时, 软件将纵墙与横墙分别 计算, 对横墙的影响系数 $\varphi$ 取 1 , 使得计算结果偏大; 后 者在按照纵墙变截面情况模拟时, 软件计算墙体的折算厚 度按居中计算, 因此折算厚度比实际折算厚度小, 使得计 算结果偏大。

\section{5. 结语}

本文以某火车站为例, 通过现场资料调查和结构检测, 对房屋的安全性能进行了结构计算分析, 结合以往此类建 筑的实践经验及结构特点, 对此类建筑的安全性鉴定提出 以下几点意见:

（1）对于实测砖强度和砂浆强度均低于规范中的情 况, 检测人员需要在软件计算中多加注意, 与工程实际情 况相符方可进行后续验算。 
（2）对于带壁柱墙的计算目前还没有计算软件可以 准确模拟, 各种建模方式均与实际计算结果存在误差, 难 以满足实际工程需要。如若粗略估算带壁柱墙的抗压承载 力建议可采用扶壁柱位置纵墙变截面的方式建模, 该方式 计算结果偏于保守且更接近于实际情况。

\section{致谢}

国家重点研发计划资助(项目编号: 2017YFC0702900)

(National Key R\&D Program of China
(No.2017YFC0702900))。

\section{参考文献}

[1] 砌体工程现场检测技术标准: GB/T50315-2011 [S]. 北京: 中国建筑工业出版社, 2011.

[2] 建筑结构检测技术标准: GB/T 50344-2019 [S]. 北京: 中 国建筑工业出版社, 2019.
[3] 砌体结构设计规范: GB50003-2012 [S]. 北京: 中国建筑 工业出版社，2011.

[4] 郭行方.既有砌体砖墙检测鉴定方法及加固对比分析[J].福 建建设科技，2020，2：5-7.

[5] 陈瑜,陆伟东,杨放.南京民国建筑的结构安全特点及抗震鉴 定研究 [J]. 建筑技术，2013,44(2):157-160.

[6] 危险房屋鉴定标准：JGJ125-2016[S].北京：中国建筑工业 出版社, 2016.

[7] 李满枝, 张小琼.上海某砌体结构厂房检测与加层加固设计 [C]. 2020年工业建筑学术交流会论文集（上册）.

[8] 马东亚, 金刚. 某砌体砌体结构加固设计 [J].建筑结构, 2019, 49 (增刊)：711-715.

[9] 房屋质量检测规程: DG/TJ 08-79-2008 [S]. 北京: 中国建 筑工业出版社, 2011.

[10] 喻云龙, 李世宏等.既有老旧房屋砂浆及砌体强度现场试验 研究 [J].建筑结构, 2019, 49（5）：23-26. 